\title{
A Distributed QoS Routing and CAC Framework: Performance Evaluation of Its SSRA and InterD Agents
}

\author{
Leonard Barolli ${ }^{\dagger}$, Makoto Ikeda $^{\ddagger}$, Arian Durresi ${ }^{\dagger \dagger}$ \\ Fatos Xhafa $^{\dagger \ddagger}$, Akio Koyama ${ }^{\ddagger \ddagger}$ \\ ${ }^{\dagger}$ Department of Information and Communication Engineering \\ Fukuoka Institute of Technology (FIT) \\ 3-30-1 Wajiro-Higashi, Higashi-ku, Fukuoka 811-0295, Japan \\ E-mail: barolli@fit.ac.jp \\ ${ }^{\ddagger}$ Graduate School of Engineering \\ Fukuoka Institute of Technology (FIT) \\ 3-30-1 Wajiro-Higashi, Higashi-ku, Fukuoka 811-0295, Japan \\ E-mail: mgm05001@ws.ipc.fit.ac.jp \\ ${ }^{\dagger}$ Department of Computer Science, Louisiana State University \\ Baton Rouge, Louisiana, USA \\ E-mail: durresi@csc.lsu.edu \\ ${ }^{\dagger \ddagger}$ Department of Languages and Informatics Systems \\ Polytechnic University of Catalonia \\ Jori Girona 1-3, 08034 Barcelona, Spain \\ E-mail: fatos@1si.upc.edu \\ ${ }^{\ddagger}$ Department of Informatics, Yamagata University \\ 4-3-16 Jonan, Yonezawa, Yamagata 992-8510, Japan \\ E-mail: akoyama@yz.yamagata-u.ac.jp
}

\begin{abstract}
In order to support multimedia communication, it is necessary to develop routing algorithms which use for routing more than one QoS parameters. This is because new services such as video on demand and remote meeting systems require better QoS. Also, for admission control of multimedia applications different QoS parameters should be considered. In our previous work, we proposed an intelligent routing and CAC strategy using cooperative agents. In this paper, we propose and evaluate the performance of SSRA algorithm and a GA-based InterD agent. Performace evaluation shows that proposed agents have a good behavior.
\end{abstract}

\section{Introduction}

The networks of today are going through a rapid evolution and they are expected to support a wide range of multimedia applications. The requirement for timely delivery of multimedia data raises new challenges for the next generation broadband networks. The key issue is the Quality of Service (QoS) routing. Also, ensuring the QoS demands to traffic flows and groups of flows is an important challenge for future broadband networks, and resource provisioning via Call Admission Control (CAC) is a key mechanism for achieving this.

The purpose of admission control is to support the QoS demands of real time applications via resource reservation. While, the purpose of QoS routing is to find good paths which satisfy user requirements.

Traditional CAC schemes can be classified in equivalent capacity, heavy traffic approximation, upper bounds of the cell loss probability, fast buffer/bandwidth allocation, and time windows. Among proposed CAC schemes, the equivalent capacity gives better results [1]. But, the equivalent capacity scheme makes many approximations, which result in an overestimate of equivalent capacity.

So far, many routing algorithms have been proposed. The routing strategies can be classified into three classes: source, distributed and hierarchical routing. Source routing algorithms are conceptually simple, but they suffer from scalability problem. Distributed routing algorithms are more scalable, but loops may occur, which make the routing to fail. Hierarchical routing has been used to cope with the scalability problems of source routing in large inter-networks. The hierarchical routing retains many advantages of source routing. It also has some advantages of distributed rout- 
ing because the routing computation is shared by many nodes. But in the conventional hierarchical routing, the network state is aggregate additional and gives some imprecision, which has a significant negative impact on QoS routing [2, 3, 4].

In order to support multimedia communication over high speed networks, it is necessary to develop routing algorithms which use for routing more than one QoS parameters such as throughput, delay, and loss probability. This is because new services such as video on demand and remote meeting systems require better QoS. However, the problem of QoS routing is difficult, because the distributed applications have very diverse QoS constraints on delay, loss ratio and bandwidth. Also, multiple constraints often make the routing problem intractable. For example, finding a feasible route with two independent path constraints is NP-complete [2].

To cope with broadband networks the CAC schemes and routing algorithms should give a fast decision and must be adaptive, flexible, and intelligent for efficient network management [5].

Use of intelligent algorithms based on Fuzzy Logic (FL), Genetic Algorithms (GA) and Neural Networks $(\mathrm{NN})$ can prove to be efficient for telecommunication networks $[6,7,8,9]$. In our previous work, we proposed an intelligent $\mathrm{CAC}$ and routing framework using cooperative agents [5]. The proposed framework is based on Distributed Artificial Intelligence (DAI) approach, which deals with design of artificial agents to develop intelligent systems. We introduced two types of agents: simple and intelligent agents. The intelligent agents were based on FL and GA. However, in the proposed Fuzzy Admission Control (FAC) scheme and Fuzzy Equivalent Capacity Estimator (FECE), we considered only two indicators for QoS and Congestion Control (CC) [10, 11]. In [12, 13], we extended our work by proposing two additional schemes: Fuzzy QoS Controller (FQC) and Fuzzy Congestion Controller (FCC), which are integrated in the previous scheme. The new scheme is called Fuzzy Admission Control for Multimedia applications (MFAC).

In [5], we proposed an Adaptive Routing method based on Genetic Algorithm (GA) (ARGA). By using a novel gene coding method, the chromosomes have the same length, which results in easy genetic operations and a fast routing decision can be achieved. Performance evaluation via simulations showed that ARGA method has a faster routing decision compared with another GA-based routing method called Genetic Load Balancing Routing (GLBR). In ARGA, we considered only time delay as the only parameter for routing. However, in order to support multimedia communication over broadband networks, it is necessary to develop routing algorithms which use for routing more than one QoS parameters.

In [14], we proposed a GA based routing algorithm which uses two QoS parameters for routing. This method called ARGAQ could support two QoS parameters: delay and transmission success rate. The simulation results showed that ARGAQ method had better performance than ARGA. However, the ARGAQ method is effective only if the parameters have a relation between them and the GA fitness function can be expressed by a mathematical formula. In $[12,13]$, we proposed a new GA-based multi-purpose optimization algorithm for QoS routing. The proposed method uses a multi-division group model for multi-purpose optimization.

In this paper, we propose and evaluate a Search Space Reduction Agent (SSRA) algorithm and a GA-based InterD agent. Per-

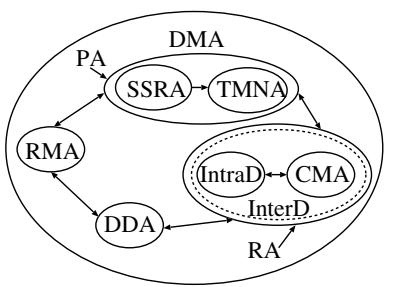

Figure 1. DMA structure.

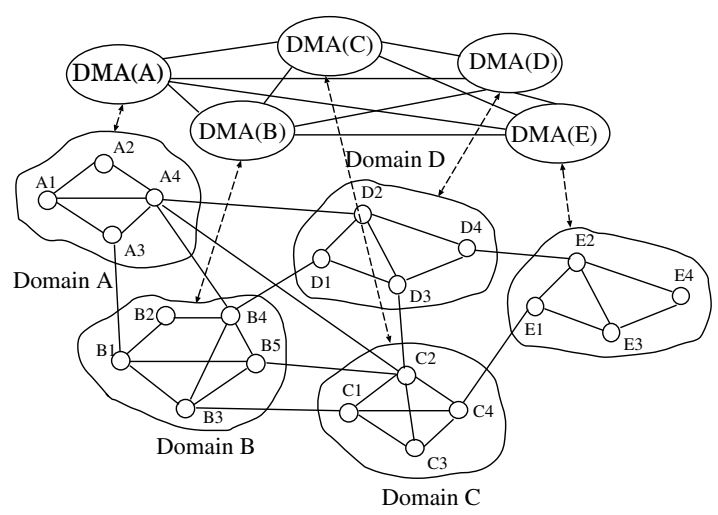

Figure 2. Distributed network architecture with DMAs.

formance evaluation shows that proposed agents have a good behavior.

The paper is organized as follows. In Section 2, we introduce the previous work. The proposed SSRA algorithms are presented in Section 3. The simulation results are discussed in Section 4. Finally, conclusions are given in Section 5.

\section{Previous Work}

\subsection{Distributed Network Architecture}

The proposed network architecture is a Multi-Agent System (MAS). The agents are distributed and cooperate together. Each Domain Management Agent (DMA) has four agents: the Resource Management Agent (RMA), Precomputation Agent (PA), which includes SSRA and Tree Model Network Agent (TMNA), Destination Discovery Agent (DDA), and Routing Agent (RA) with its Intra Domain (IntraD) and Inter Domain (InterD) agents. The DMA structure is shown in Fig.1. The PA includes SSRA and TMNA. We call these two agents PA, because they make the computation before the RA is activated. The computation time starts when a new connection makes a request to the network. The RA has the IntraD and InterD agents. In fact, the InterD agent is a composition of IntraD agent and Connectivity Management Agent (CMA), which are activated by an escalation strategy. The distributed network architecture with DMAs is shown in Fig.2. This architecture can be considered as a hierarchical architecture, where in first level are domains and in the second level are DMAs. We have shown here only five domains. But, this architecture can be scaled-up easily by increasing the number of DMAs and domains in order to deal with the increasing users demands and number of switches. 


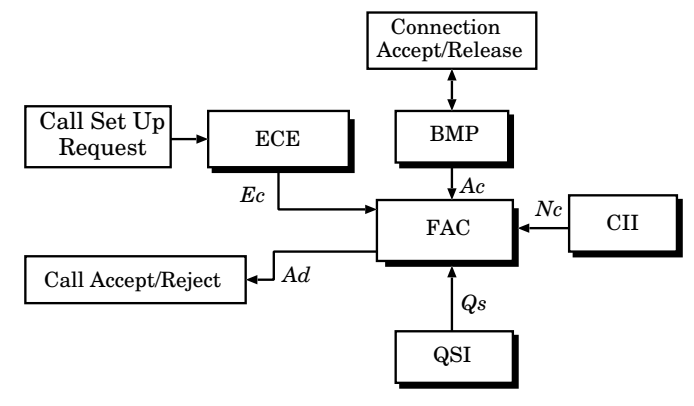

Figure 3. FAC scheme.

\subsection{RMA}

The proposed RMA is based on FL [15] In difference from the equivalent capacity admission control method [1], which uses only the available capacity as the only variable for the call admission decision, our FAC scheme considers four parameters: Quality of service $(Q s)$, Network congestion parameter $(N c)$, Available capacity $(A c)$, and user requirement parameter which is expressed by Equivalent capacity $(E c)$. The output linguistic parameter is the Acceptance decision $(A d)$. In order to have a soft admission decision, not only "accept" and "reject" but also "weak accept", "weak reject", and "not accept not reject" are used to describe the accept/reject decision.

The FAC scheme is shown in Fig.3. The information for FAC are given by Bandwidth Management Predictor (BMP); Congestion Information Indicator (CII); Quality of Service Indicator (QSI); and Equivalent Capacity Estimator (ECE). The BMP works in this way: if a connection is accepted, the connection bandwidth is subtracted from the available capacity of the network, otherwise, if a connection is released, the connection bandwidth is added to the available capacity of the network. The CII decides whether the network is or isn't congested. The QSI determines whether allowing a new connection violates or not the QoS guarantee of the existing connections. In order to get a better estimation of $E c$, we proposed a Fuzzy ECE (FECE) scheme. But, for the sake of the space, we will not explain here (for more explanation see [10]).

\subsection{PA and DDA}

\subsubsection{SSRA}

By using the SSRA a network with many nodes and links will be reduced in a network with a small number of nodes and links. Thus, the proposed framework is able to cope with more largescale networks.

\subsubsection{TMNA}

After the execution of SSRA, the effective topology of the network is transformed in a tree model by TMNA. To explain this procedure, a small network with 8 nodes as shown in Fig. 4 is considered. Node A is the Source Node ( $\mathrm{SN}$ ) and node $\mathrm{H}$ is the Destination Node (DN). All paths are expressed by the tree model shown in Fig.5. In the shaded areas are shown the same paths from node

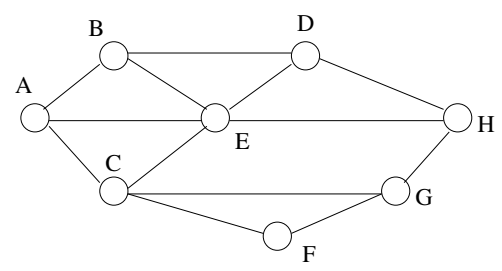

Figure 4. A network example with 8 nodes.

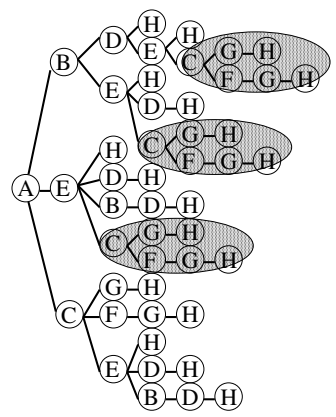

Figure 5. Network tree model.

$\mathrm{C}$ to $\mathrm{H}$. Therefore, we further reduce the tree network as shown in Fig.6. The tree model constructed by TMNA is used by IntraD agent for intra-domain routing. In the reduced tree model, each tree junction is considered as a gene and the path is represented by the chromosome. In this case, two tree junctions were omitted, because they represent the same routes from node $\mathrm{C}$ to $\mathrm{H}$.

\subsubsection{DDA}

After a new connection is accepted, the RMA sends a request to the DDA. The DDA consults a table with node name entries to check whether SN and DN are in the same domain or not. If SN and DN are in the same domain, the DDA of the source domain activates the IntraD agent. Otherwise, if the $\mathrm{SN}$ and $\mathrm{DN}$ are in different domains, the InterD agent is activated.

\subsection{IntraD Agent}

The IntraD algorithm is a delay-constraint unicast source routing mechanism and is based on GA [16]. The most important factor to achieve efficient genetic operations is gene coding. In the GLBR algorithm [6], the genes are put in a chromosome in the same order the nodes are in a path, so the chromosomes have different sizes which result in complex crossover operation. In order to simplify the genetic operations of GLBR, in the IntraD algorithm, the network is expressed by a tree network and the genes represent the tree junctions. A chromosome example is shown in Fig.7. The genes in a chromosome have two states "active" and "inactive". A gene is called "active" if the junction is in the path, otherwise the gene is in "inactive" state. The genetic operations are carried out in the "active" genes. Each gene includes information of the adjacent nodes. The paths are represented by chromosomes which have the same length. Therefore, the crossover operation becomes easy. 


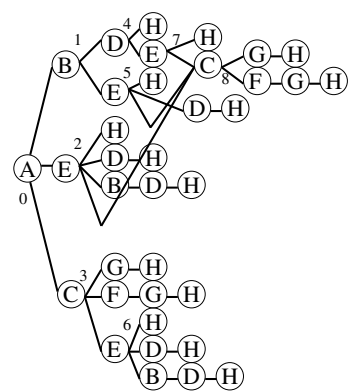

Figure 6. Reduced network tree model.

\begin{tabular}{c|c|cccccc|c|c|c|c|}
0 & 1 & 2 & 3 & 4 & 5 & 6 & 7 & 8 \\
\hline BEC & DEE & H D & GFE & H E & HDC & HDB & H C & G F \\
\hline
\end{tabular}

Figure 7. Gene coding.

\subsection{InterD Agent}

After the DDA finds out that SN and DN are in different domains, the InterD agent is activated. The InterD agent is a composition of IntraD agent and CMA. It uses an escalation strategy to make the inter-domain routing. By using the escalation strategy, the information exchange is needed only in domains where the selected path passes. Thus, the information flooding in all domains is not necessary and the network resources can be used efficiently. The InterD agent operates in the following way. After receiving a connection request, a node become a SN. The IntraD agent finds a path inside the domain. The DN of the source domain starts the CMA. The CMA is a simple agent. It finds the best link by using a sorting algorithm based on the inter-domain links parameters. After the CMA decides the best link for connection, the DN of this link becomes a $\mathrm{SN}$ and the IntraD agent is activated in the following domain. This procedure is escalated until the $\mathrm{DN}$ of the destination domain is found.

\subsection{MFAC Scheme}

In our previous work, we considered only two indicators for QoS and CC. However, for multimedia applications more QoS and $\mathrm{CC}$ parameters should be considered. For this reason, we designed two fuzzy based controllers: FQC and FCC. The scheme of proposed MFAC is shown in Fig. 8.

\subsubsection{FQC and FCC}

As input linguistic parameters for FQC, we consider the throughput $T h$, the delay $D$, and the loss probability $L p$. The term sets of $T h, D$, and $L p$ are defined respectively as:

$$
\begin{aligned}
T(T h) & =\{\text { Small, Medium, Large }\}=\{\text { Sa, Mu, Lr }\} \\
T(D) & =\{\text { Low, Middle, High }\}=\{L o, M i, H i\} \\
T(L p) & =\{\text { Low, Normal, High }\}=\{L w, N r, H g\} .
\end{aligned}
$$

From our experience, we decided that three membership functions are enough for $T h$ linguistic parameter, three membership functions are enough for $D$ linguistic parameter, and two membership functions are enough for $L p$ linguistic parameter.

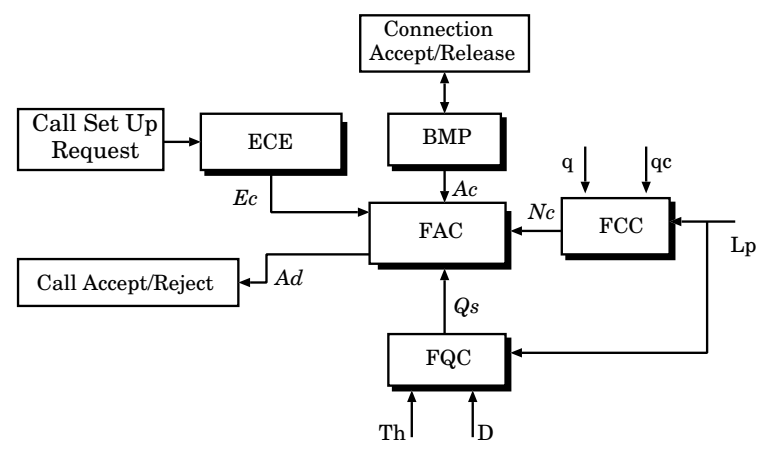

Figure 8. Proposed MFAC scheme.

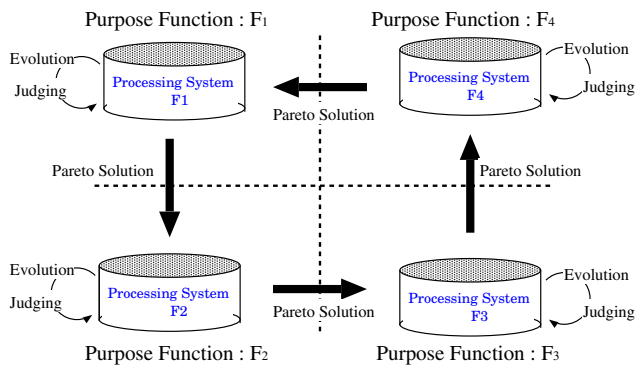

Figure 9. Multiple-purpose optimization.

The term set of the output linguistic parameter $T(Q s)$ is defined as $\{$ Not Satisfied, Weak Satisfied, Normal, Not So Satisfied, Satisfied $\}$. We write for short as $\{$ NS, WS, N, NSS, SA $\}$.

For FCC, as input linguistic parameters, we consider the queue length $Q l$, the queue length change rate $Q c r$, and the loss probability $L p$. The term sets of $Q l, Q c r$, and $L p$ are defined respectively as:

$$
\begin{aligned}
T(Q l) & =\{\text { Empty, Middle, Full }\}=\{E, M i, F u\} \\
T(Q c r) & =\{\text { Negative, Positive }\}=\{\text { Ne,Po }\} \\
T(L p) & =\{\text { Low, Normal, High }\}=\{L w, N r, H g\} .
\end{aligned}
$$

Usually for congestion control is used a two threshold congestion method. In this method, the system is considered congested if the queue length exceeds the high threshold and un-congested if the queue length drops below the low threshold. For this reason, the maximum value of $Q l$ would be the total buffer size. The edges of the membership functions can be the low and high threshold. For the $Q c r$ linguistic parameter the maximum positive and negative queue length change would be the queue length. For the $L p$ linguistic parameter, the values for "Low", "Normal" and "High" can be decided considering the required QoS.

The term set of the output linguistic parameter $T(C s)$ is defined as $\{$ Decrease More, Decrease Slightly, No Congestion, Increase Slightly, Increase More $\}$. We write for short as $\{\mathrm{DM}, \mathrm{DS}$, NC, IS, IM\}.

\subsection{Multi-objective Optimization}

The proposed method uses the multi-division group model for multi-objective optimization. When a function can be divided in different objective functions, the global domain can be divided in different domains and each individual can evolve in its domain. 


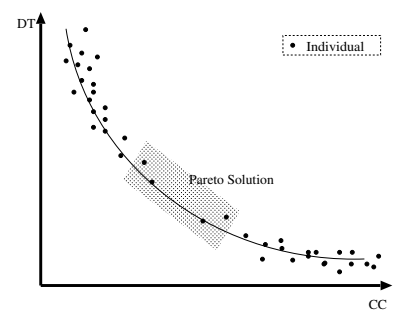

Figure 10. Pareto solution for DT and CC.

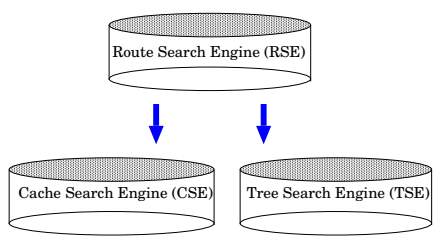

Figure 11. RSE structure.

The procedure is shown in Fig.9, where four different objective functions are independent from each other and each process is operating independently in each domain. In Fig.10 is shown an example of Delay Time (DT) and Communication Cost (CC). The vertical axis shows the DT and horizontal axis the $\mathrm{CC}$. The DT and $\mathrm{CC}$ have trade-off relations. The points in the figure show the individuals (routes). The individuals which are in the left-upper part of the figure have the lowest CC. On the other hand, the individuals which are in the right-lower part of the figure have the lowest DT values. The individuals which are in the shaded area have good values for both DT and CC. The shaded area is called "pareto solution". The individuals near pareto solution can be found by exchange the solutions of different domains.

\subsubsection{QoS Routing Search Engine}

The structure of Routing Search Engine (RSE) is shown in Fig.11. It includes two search engines: Cache Search Engine (CSE) and Tree Search Engine (TSE). Both engines operate independently, but they cooperate together to update the route information. When the RSE receives a request for QoS routing from a client, it forwards the request in parallel to CSE and TSE. Then, the CSE and TSE search in parallel to find a route satisfying the required QoS. The CSE searches for a route in the cache database (in the cache database, the destination and route information is saved as a database item). If the found route by CSE satisfies the required QoS, this route information is sent to RSE, otherwise the route is put in the gene pool as new individual. If a QoS route can not be found by CSE, the route found by TSE is sent to RSE. It should be noted that CSE is faster than TSE, because the TSE searches for all routes in its domain using a GA-based routing.

\subsubsection{CSE-TSE Cooperation and Database Up- dating}

The database information should be updated because the network traffic and the network state change dynamically. In order to update the database, the CSE and TSE cooperate together. When the TSE finds a good QoS route, it puts this route information in the cache database as shown in Fig.12. The route information

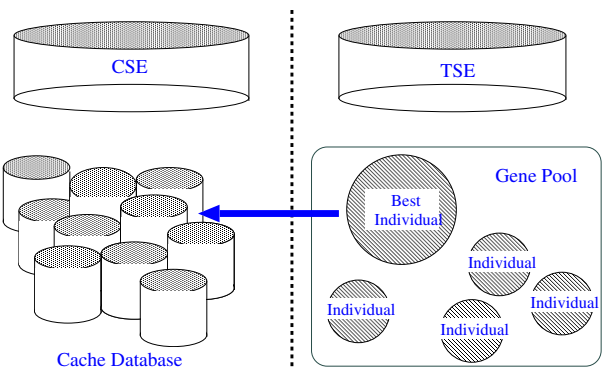

Figure 12. CSE and TSE cooperation1.

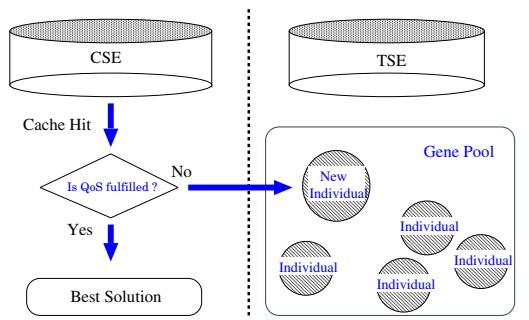

Figure 13. CSE and TSE cooperation2.

which is used frequently is given high priority, thus this route can be searched very fast by CSE. In the case when the CSE finds a route in the database, but this route does not fulfill the required QoS by client, this route information is put as a new individual in the genepool of TSE as shown in Fig.13 and will be used as a new individual during the selection process of the GA.

\section{Proposed SSRA Algorithm}

The flowchart of SSRA is shown in Fig.14. The key element of SSRA is Effective Topology (ET) extraction. In order to extract the ET, the network connectivity information, link and node metrics, and QoS requirement of the new connection are required. In our previous work [12], we used link parameters $T S R \_T h, D T \_T h, C C_{-} T h$ to specify the QoS demands of a new connection. If $D T<D T \_T h, T S R>T S R \_T h, C C<$ $C C \_T h$ for a connection, this means that every path which passes via this link satisfies the connection requirements.

In different from [12], in this work we consider the link and node metrics. We assume 4 QoS parameters and if the node metric is zero it means that the proposed SSRA algorithm is the same with algorithm in [12]. We change the number of link and node parameters to see what kind of effect they have on the network topology.

First, the SSRA based on the required QoS parameters checks all links in the network whether they satisfy or not the threshold parameters. If a link doesn't satisfy the threshold parameters, it is excluded from ET. Otherwise, the link is included in the ET and the next link is checked. The procedure is repeated until all links are finished. Then, the algorithm checks all the nodes if they satisfy the required parameters. After all links and nodes are checked, the network ET is constructed and the complete procedure is finished. 


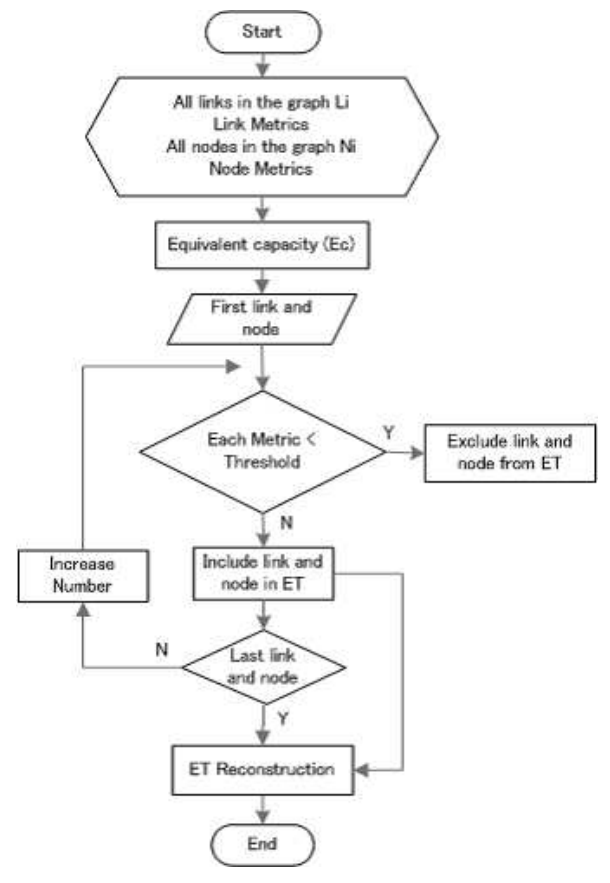

Figure 14. SSRA flowchart.

\section{Simulation Results}

\subsection{Simulation Results for SSRA}

The simulation results of SSRA in the case when we consider only the link parameters are shown in Fig.15. For the simulation, we used a computer with these specifications: (OS: Windows XP Pro; IDE: Visual C++6.0; CPU: Athlon64 +3500, Memory: 1024MB). The QoS parameters were set up in a random way. The DT for each link was set between 1 and 100, TSR was set between 1 and $100 \%$ and CC between 1 and 200 units.

In Fig.15, we considered $T S R_{-} T h=50, C C_{-} T h=150$ and changed DT_Th from 25 to 100 . As shown in the figure with the increase of the DT threshold the success rate is increased. This is because more number of links will be available. However, when the threshold is very small the success rate is less than $20 \%$. Therefore, there are trade-offs for deciding the threshold parameters.

In Fig.16 and Fig.17 are shown the effects of the number of nodes and node parameters on the algorithm processing time and remaining number of links, respectively. In Fig.16, with the increase of number of nodes, the processing time is increased. However, we see that the number of node parameters has not a big effect on the processing time. The same behavior is also shown in Fig.17. With the increase of number of nodes, we have more links in the effective topology of the network. Based on these results, we can find the tradeoff parameters for different kind of networks.

In Fig.18, we carried out the simulation for a threshold value 25 units. While in Fig.19, we increased the threshold value to 30 units. These results shows that by increasing the threshold value, both the remaining number of links and the processing time is decreased.

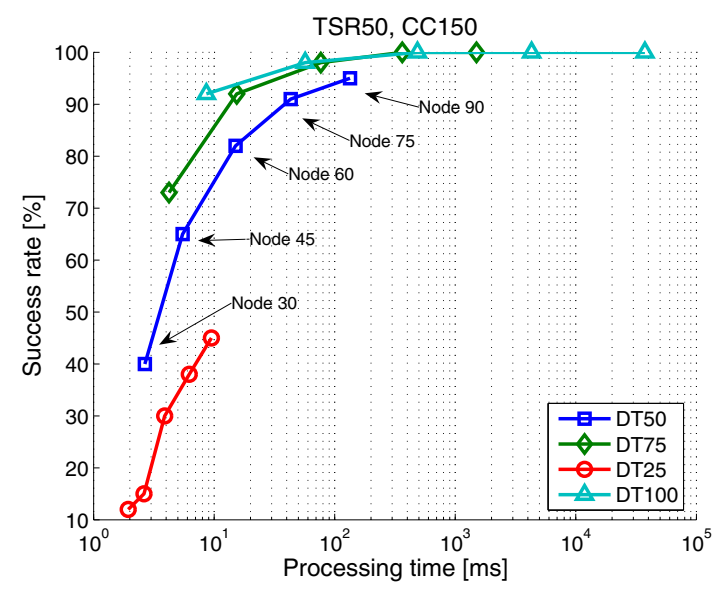

Figure 15. Success rate versus processing time.

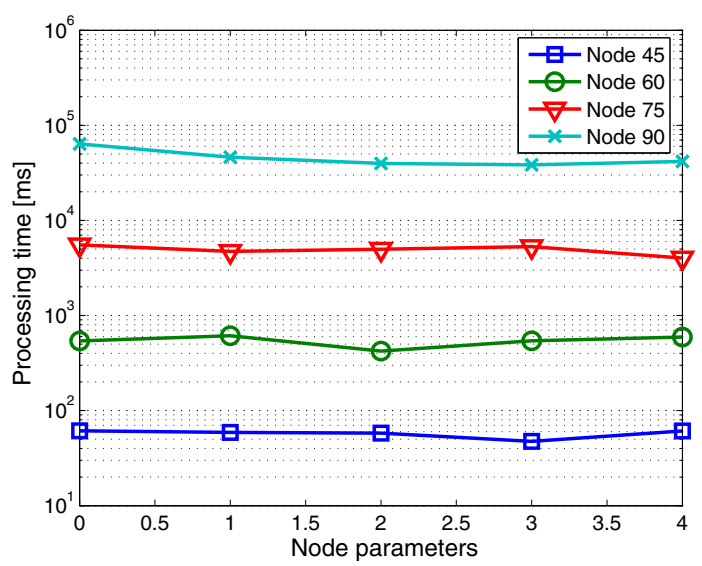

Figure 16. Processing time versus node parameters.

\subsection{InterD Agent}

For the simulation, we use a network with 20 nodes. The network model is shown in Fig.20. We consider that the network is reduced by SSRA. First, we set in a random way the QoS requirements. The RSE generates in random way the values of the required QoS and the destination node information. Next, the CSE and TSE search in parallel to find a route. If the CSE finds a route in the cache database it checks whether this route satisfies the QoS or not. If the found route satisfies the required QoS is sent back to the RSE. Otherwise, the route is put as a new individual in the gene pool. If a QoS route can not be found by CSE, the route found by TSE is sent to RSE. The genetic operations are repeated until a solution is found or the number of 200 generations is achieved. Then, the selected route is put in the cache database.

In Fig. 21 and Fig. 22 are shown the simulation results using TSE. In Fig. 21, we show the Generation Size (GS) versus the number of individuals. While, in Fig.22 is shown the processing time versus the number of individuals. We consider 4 cases when 


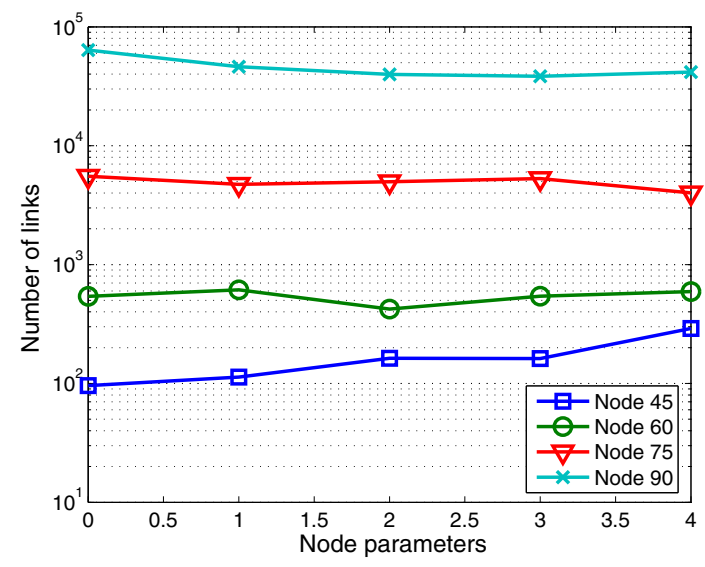

Figure 17. Number of links versus node parameters.

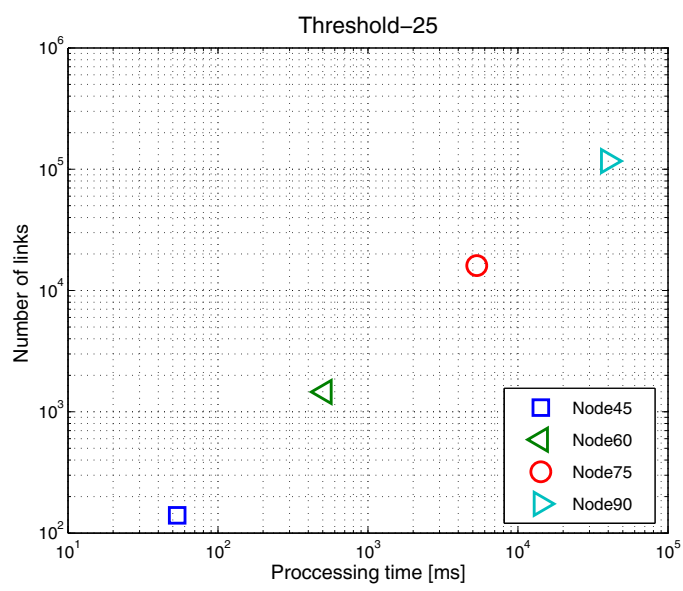

Figure 18. Algorithm performance for threshold value 25 units.

the number of exchanging individuals is $3,5,7$, and 10 , respectively.

If there are few individuals in the population, the GS which shows the number of generations needed to find a solution becomes large. On the other hand, when the number of individuals is high, the GS to find a solution becomes small. However, when the number of individuals is 12 and 16, the difference is very small. This is because some individuals become the same in the genepool.

Considering the exchange of individuals between domains, it can be seen that when the exchange interval is short the solution can be found very fast. This shows that by exchanging the individuals the algorithm can approach very quickly to the pareto solution.

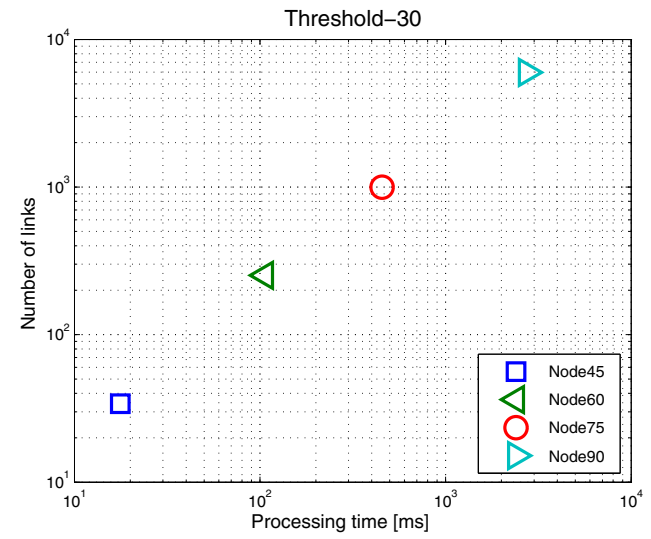

Figure 19. Algorithm performance for threshold value 30 units.

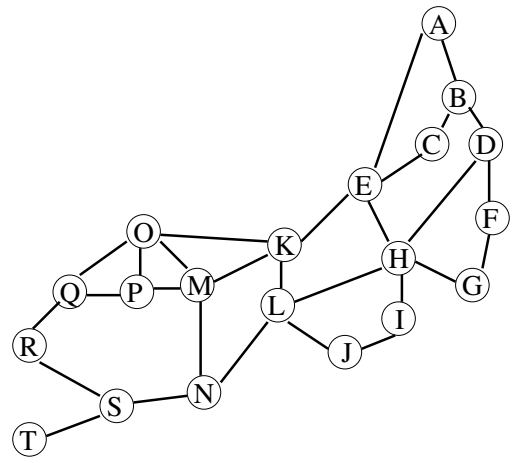

Figure 20. Network model with 20 nodes.

\section{Conclusions}

In this paper, we proposed and evaluated the performance of SSRA algorithm and a GA-based InterD agent. From the simulation results, we conclude:

- the proposed method can support QoS routing for multiple metrics;

- the number of node parameters has not a big effect on the processing time;

- with the increase of the number of nodes, we have more links in the effective topology of the network;

- by increasing the threshold values, both the remaining number of links and the processing time is decreased;

- if there are few individuals in the population, the GS becomes large;

- when the number of individuals is high, the GS to find a solution becomes small;

- when the number of individuals is 12 and 16 , the difference is very small. This is because some individuals become the same in the genepool; 


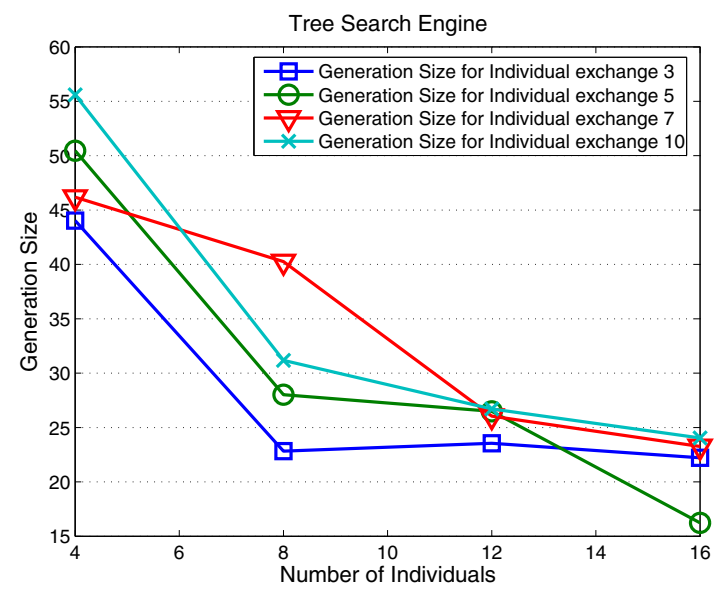

Figure 21. Generation size versus number of individuals.

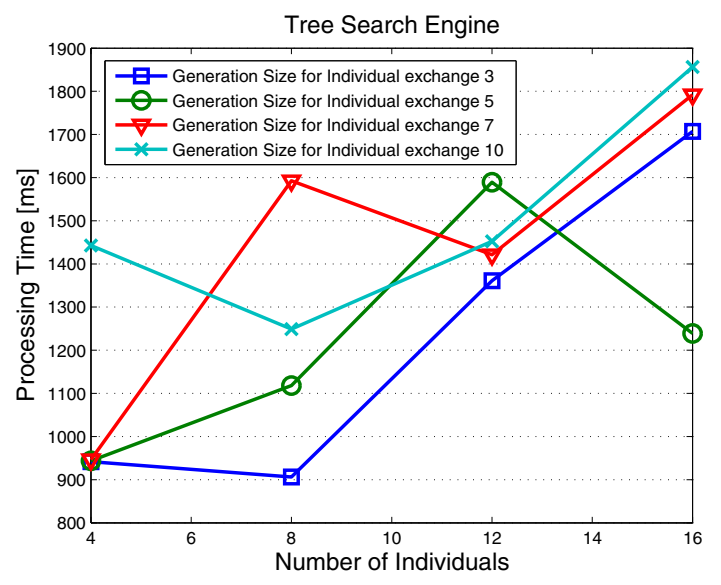

Figure 22. Processing time versus number of individuals.

- when the exchange interval is short the solution can be found very fast. This shows that by exchanging the individuals the algorithm can approach very quickly to the pareto solution.

In the future, we would like to carry out extensive simulations to evaluate the overall performance of the proposed framework.

\section{References}

[1] R.Guérin, H.Ahmadi, and M.Naghshineh, "Equivalent Capacity and Its Application to Bandwidth Allocation in HighSpeed Networks", IEEE J. Select. Areas Commun., Vol.9, No.7, pp.968-981, 1991.

[2] S.Chen and K.Nahrstedt, "An Overview of Quality of Service Routing for Next-Generation High-Speed Networks: Problems and Solutions", IEEE Network, Special Issue on Transmission and Distribution of Digital Video, Vol.12, No.6, pp.64-79, 1998.
[3] C. Baransel, W. Dobosiewicz and P.Gburzynski, "Routing in Multihop Packet Switching Networks: Gb/s Challenge", IEEE Network, Vol.9, No.3, pp.38-60, 1995.

[4] W.C.Lee, M.G.Hluchyj and P.A.Humblet, "Routing Subject to Quality of Services Constraints in Integrated Communications Networks", IEEE Network, Vol.9, No.4, pp.46-55, 1995.

[5] L.Barolli, A.Koyama, T.Yamada, S.Yokoyama, T.Suganuma and N.Shiratori, "An Intelligent Routing and CAC Framework for Large Scale Networks Based on Cooperative Agents", Computer Communications International Journal, Vol.25, No.16, pp.1429-1442, October 2002.

[6] M.Munetomo, Y.Takai and Y.Sato, "An Adaptive Routing Algorithm with Load Balancing by a Genetic Algorithm", Trans. of IPSJ, Vol.39, No.2, pp.219-227, 1998.

[7] L. Barolli, A. Koyama, S.Motegi and S.Yokoyama, "Performance Evaluation of a Genetic Algorithm based Routing Method for High-speed Networks", Trans. of IEE Japan, Vol.119-C, No.5, pp.624-631, May 1999.

[8] L.Barolli, A.Koyama, T.Yamada, and S.Yokoyama, "An Intelligent Policing-Routing Mechanism Based on Fuzzy Logic and Genetic Algorithms and Its Performance Evaluation", IPSJ Journal, Vol.41, No.11, pp.3046-3059, November 2000.

[9] I. Habib (Ed.), "Neurocomputing in High-speed Networks", IEEE Commun. Magazine, Special Issue, Vol.33 No.10, 1995.

[10] L.Barolli, A.Koyama, T.Yamada, S.Yokoyama, T.Suganuma, and N.Shiratori, "A Fuzzy Based Equivalent Capacity Estimation Method for Bandwidth Allocation in High-Speed Networks", IPSJ Journal, Vol.42, No.8, pp.2167-2175, 2001.

[11] L.Barolli, A.Koyama, T.Yamada, S.Yokoyama, T.Suganuma and N.Shiratori, "A Fuzzy Admission Control Scheme and Its Performance Evaluation”, IPSJ Journal, Vol.42, No.12, pp.3213-3221, December 2001.

[12] M. Ikeda, L. Barolli, G. De Marco, A. Durresi, A. Koyama, M. Durresi, "Evaluation of a Network Extraction Topology Algorithm for Reducing Search Space of a GA-based Routing Approach", Proc. of MNSA-2006/ICDCS-2006, Lisbon, Portugal, CD-ROM Proceedings, 6 pages, July 2006.

[13] M. Ikeda, L. Barolli, A. Koyama, A. Durresi, G. De Marco, J. Iwashige, "Performance Evaluation of an Intelligent CAC and Routing Framework for Multimedia Applications in Broadband Networks", Journal of Computer and System Science (JCSS), Vol. 72, Issue 7, pp. 1183-1200, November 2006.

[14] L.Barolli, A.Koyama, T.Suganuma and N.Shiratori, "A Genetic Algorithm Based QoS Routing Method for Multimedia Communications Over High-Speed Networks", IPSJ Journal, Vol.44, No.2, pp.544-552, February 2003.

[15] D.Dubois, H.Prade, and R.Yager, (Eds.), Fuzzy Sets for Intelligent Systems, Morgan Kaufman Publishers, 1993.

[16] D.E.Goldberg, Genetic Algorithms in Search, Optimization, and Machine Learning, Addison-Wesley, 1989. 